\title{
Phase II study of multidisciplinary therapy combined with pembrolizumab for patients with synchronous oligometastatic non- small cell lung cancer TRAP OLIGO study (WJOG11118L)
}

Taichi Miyawaki ${ }^{1,2}$, Hirotsugu Kenmotsu $^{1^{*}}$ (D), Hideyuki Harada ${ }^{3}$, Yasuhisa Ohde ${ }^{4}$, Yasutaka Chiba ${ }^{5}, K_{\text {Koji Haratani }}{ }^{6}$, Tamio Okimoto ${ }^{7}$, Tomohiro Sakamoto ${ }^{8}$, Kazushige Wakuda ${ }^{1}$, Kentaro Ito ${ }^{9}$, Takehiro Uemura ${ }^{10}$, Shinya Sakata ${ }^{11}$, Yoshihito Kogure $^{12}$, Yasumasa Nishimura ${ }^{13}$, Kazuhiko Nakagawa ${ }^{6}$ and Nobuyuki Yamamoto ${ }^{14}$

\begin{abstract}
Background: Synchronous oligometastatic non-small cell lung cancer (NSCLC) is generally characterised by the limited number of metastases at the time of diagnosis. Several clinical trials have shown that local ablative therapy (LAT) at all sites of the disease might be beneficial for patients with oligometastatic NSCLC. In recent years, the combination of programmed cell death 1 (PD-1) inhibitors or programmed cell death ligand 1 with cytotoxic chemotherapy has become a new standard treatment for patients with metastatic NSCLC. Furthermore, multisite LAT would inherently reduce the overall tumour burden, and this could promote $T$ cell reinvigoration to enhance the efficacy of PD-1 inhibitors. Few studies have evaluated the efficacy of the combination of PD-1 inhibitors with LAT at all sites of disease. The aim of the present multicentre single-arm phase II study is to evaluate the efficacy of LAT at all sites of disease following standard platinum doublet chemotherapy with pembrolizumab in patients with oligometastatic NSCLC.

Methods: Thirty patients with synchronous oligometastatic NSCLC will be enrolled in the trial. All patients will receive 2-4 cycles of a systemic treatment including pembrolizumab and chemotherapy as induction therapy. Patients who will receive LAT will be determined by a multidisciplinary tumour board, including medical oncologists, radiation oncologists, and thoracic surgeons. LAT will be administered at all sites of disease within 2156 days of the last dose of induction therapy and will be followed by maintenance therapy within 42 days of the last day of LAT. The primary endpoint is the progression-free survival (PFS) rate of 24 months from the date of initiation of LAT. The secondary endpoints are toxicity, response to induction therapy, PFS, overall survival, and the frequency of LAT.
\end{abstract}

\footnotetext{
* Correspondence: h.kenmotsu@scchr.jp

${ }^{1}$ Division of Thoracic Oncology, Shizuoka Cancer Center, 1007

Shimonagakubo, Nagaizumi-cho, Sunto-gun, Shizuoka 411-8777, Japan

Full list of author information is available at the end of the article
}

(C) The Author(s). 2021 Open Access This article is licensed under a Creative Commons Attribution 4.0 International License, which permits use, sharing, adaptation, distribution and reproduction in any medium or format, as long as you give appropriate credit to the original author(s) and the source, provide a link to the Creative Commons licence, and indicate if changes were made. The images or other third party material in this article are included in the article's Creative Commons. licence, unless indicated otherwise in a credit line to the material. If material is not included in the article's Creative Commons licence and your intended use is not permitted by statutory regulation or exceeds the permitted use, you will need to obtain permission directly from the copyright holder. To view a copy of this licence, visit http://creativecommons.org/licenses/by/4.0/ The Creative Commons Public Domain Dedication waiver (http://creativecommons.org/publicdomain/zero/1.0/) applies to the data made available in this article, unless otherwise stated in a credit line to the data. 


\section{Discussion: This study will provide novel data on the efficacy and safety profile of the combination of LAT and chemotherapy plus immune-checkpoint inhibitors in patients with synchronous oligometastatic NSCLC. If the primary endpoint of this study is met, extensive phase III studies further assessing this strategy will be recommended.}

Trial registration: jRCT identifier: jRCTs041200046 (date of initial registration: 28 October 2020).

Keywords: Clinical trial, Programmed cell death 1 inhibitor, Oligometastatic disease, Local ablative therapy, Progression-free survival

\section{Background}

Synchronous oligometastatic non-small cell lung cancer (NSCLC) is generally characterised by the limited number of metastases at the time of diagnosis [1]. In recent years, oligometastatic NSCLC has been defined as a disease stage harbouring few metastases treatable with local ablative therapies (LAT). Predominant patterns of initial progressive disease (PD) after first-line systemic therapy suggested the utility of LAT for all sites in patients with oligometastatic NSCLC. Several prospective trials for patients with oligometastatic NSCLC suggested that LAT might be beneficial [2, 3]. Furthermore, a randomised phase II trial in patients with oligometastatic NSCLC (1-3 metastases) has shown that LAT at all sites of the disease is associated with a significant improvement in overall survival (OS) and progression-free survival (PFS) compared with maintenance therapy alone $[4,5]$. Another randomised phase II trial in oligometastatic NSCL C patients with 1-5 metastases also showed that LAT to all sites of disease after systemic therapy is associated with a significant improvement in PFS compared with maintenance therapy alone [6]. In both trials, cytotoxic chemotherapy or targeted therapy could only be administered as systemic therapy. In recent years, programmed cell death 1 (PD-1) inhibitors have revolutionised the treatment of NSCLC. The KEYNOTE-189 and KEYNOTE-407 studies showed that the addition of pembrolizumab to platinum doublet chemotherapy improved OS in patients with non-squamous and squamous NSCLC, regardless of programmed cell death ligand 1 expression [7, 8].

Some studies suggested that tumour burden was correlated with exhausted CD8 T-cells, which is a negative prognostic indicator for anti-PD-1 inhibitors $[9,10]$. Furthermore, pre-clinical studies suggested that tumour debulking improved treatment outcomes [11]. LAT to all sites of disease that reduce tumour burden as much as possible might maximise synergy effects with PD-1 inhibitors in patients with oligometastatic NSCLC. In fact, the phase II trial of pembrolizumab after LAT for oligometastatic NSCLC showed promising results with a twoyear OS of $77.5 \%$ [12]. However, the optimal treatment for patients with untreated synchronous oligometastatic
NSCLC remains unclear because $70 \%$ of the participants in the previously mentioned study were patients with metachronous oligometastatic NSCLC, and over 50\% of the participants were previously treated with systemic therapy [12]. Thus, we are conducting a multicentre single-arm phase II study to evaluate the efficacy of LAT at all disease sites following therapy with a combination of pembrolizumab and platinum doublet for patients with untreated synchronous oligometastatic NSCLC.

\section{Patients and methods \\ Participants}

Planned enrolment period is 2020 October to 2022 October, and the observation period will be 3 -year followup period from the time the last patient is enrolled. Key inclusion and exclusion criteria are shown in Table 1. Patients with an activating driver mutation (epidermal growth factor receptor [EGFR], anaplastic lymphoma kinase $[A L K]$, proto-oncogene 1 [ROS1], BRAF and $M E T)$ are not eligible for this study because the initial standard treatment for these population is different. The protocol will be amended to exclude patients with new potential activating driver mutation if approved during the period of this study. Furthermore, multiplex gene tests are not applicable to all patients in clinical practice. Therefore, in some patients, the single-plex gene test will be used to evaluate a few driver mutations such as EGFR or $A L K$. Potential participants will be enrolled in this study after receiving a full explanation and obtaining consent of the study.

Participating institutions include public hospitals, university hospitals, and cancer centers in Japan. The institutions in the study are; Hokkaido University Hospital, Sendai Kousei Hospita, Niigata Cancer Center Hospital, Juntendo University Hospital, Kanagawa Cardiovascular and Respiratory Center, Nagoya City University Hospital, Aichi Cancer Center Hospital, Aichi Medical University Hospital, Kindai University Hospital, Kansai Medical University Hospital, Kishiwada City Hospital, Osaka City General Hospital, Kobe City Medical Center General Hospital, Kurume University Hospital, Kyushu University Hospital, National Hospital Organization Kyusyu Cancer Center, 
Table 1 Key Inclusion and Exclusion Criteria

Inclusion criteria
1. Age $\geq 20$ years, < 75 years
2. Written informed consent
3. Histologically or cytologically confirmed NSCLC
4. Activating driver mutation negative or unknown: epidermal growth
factor receptor (EGFR), anaplastic lymphoma kinase (ALK), proto-
oncogene 1 (ROS1), BRAF and MET
5. No previous chemotherapy
6. Synchronous oligo-metastatic stage IV disease: maximum of three dis-
tant metastases
7. Suitable candidate for LAT (radiotherapy and/or surgery) to all site of
disease, as determined by a multidisciplinary tumour board
8. Eastern Cooperative Oncology Group (ECOG) performance status
score of 0 or 1
9. No malignant dissemination, pericardial effusion, leptomeningeal
metastases, peritoneal dissemination and ascites
10. Adequate hematologic and organ function
Exclusion criteria
1. Presence of any other active cancer
2. Presence of active infections requiring antibiotics
3. History of active autoimmune disease requiring systemic treatment
4. History of interstitial lung disease diagnosed or severe chronic
obstructive pulmonary disease (COPD)
aERR/ALKROS-1/BRAFIMET testing is not mandatory for patents wht

${ }^{a}$ EGFR/ALK/ROS-1/BRAF/MET testing is not mandatory for patients with squamous carcinoma

b Based on a previous study, any metastatic thoracic lymph nodes (N1-N3), including in the supraclavicular fossae, were collectively considered a single metastasis

Kumamoto University Hospital and Shizuoka Caner Center.

\section{Study design}

This study was approved by the central review board of Shizuoka Cancer center (number: 20-1-20-1). The study is registered in the Japan registry of Clinical Trials (jRCTs041200046) and conducted in compliance with the Declaration of Helsinki. Because of the lack of definitive data on efficacy and safety, this study was conducted as an exploratory single-arm phase II trial. Should this study achieve its primary endpoint, we will conduct a phase III trial to evaluate the effect of adding LAT to platinum-doublet chemotherapy plus pembrolizumab for patients with synchronous oligometastatic NSCLC.

In this single-arm phase II trial, we plan to enrol patients with synchronous oligometastatic NSCLC at diagnosis (defined as $\leq 3$ metastases, according to previous trials and the current trial) [4,5]. Figure 1 shows the study schema. The Protocol-prescribed treatment will continue until disease progression or met discontinuation criteria for up to 2 years.

All patients will receive $2-4$ cycles of systemic treatment as induction therapy. Patients with non-squamous histology will receive pembrolizumab (200 $\mathrm{mg} /$ body $)+$ cisplatin $\left(75 \mathrm{mg} / \mathrm{m}^{2}\right)+$ pemetrexed $\left(500 \mathrm{mg} / \mathrm{m}^{2}\right)$, or pembrolizumab (200 mg/body) + carboplatin (area under the curve [AUC] 5$)+$ pemetrexed $\left(500 \mathrm{mg} / \mathrm{m}^{2}\right)$ by intravenous infusion on the first day. Patients with squamous histology will receive pembrolizumab (200 $\mathrm{mg} /$ body $)+$ carboplatin (AUC 6) on the first day and nab-paclitaxel $\left(100 \mathrm{mg} / \mathrm{m}^{2}\right)$ on days 1,8 , and 15 , or pembrolizumab (200 mg/body) + carboplatin (AUC 6) + paclitaxel (200 $\mathrm{mg} / \mathrm{m}^{2}$ ) by intravenous infusion on the first day. This treatment, defined as ' 1 cycle', will be repeated every 3 weeks until 4 cycles.

Patients will receive LAT at all sites of disease within 21-56 days of the last dose of induction therapy. Patients who will receive LAT will be determined by a multidisciplinary tumour board (including medical oncologists, radiation oncologists, and thoracic surgeons). LAT eligibility will be evaluated based on the comorbidity of the

\section{Single-arm Phase II study}

Phase II Study of Multidisciplinary Therapy Combined with Pembrolizumab for Patients with Synchronous Oligometastatic Non-Small Cell Lung Cancer TRAP OLIGO study (WJOG11118L)

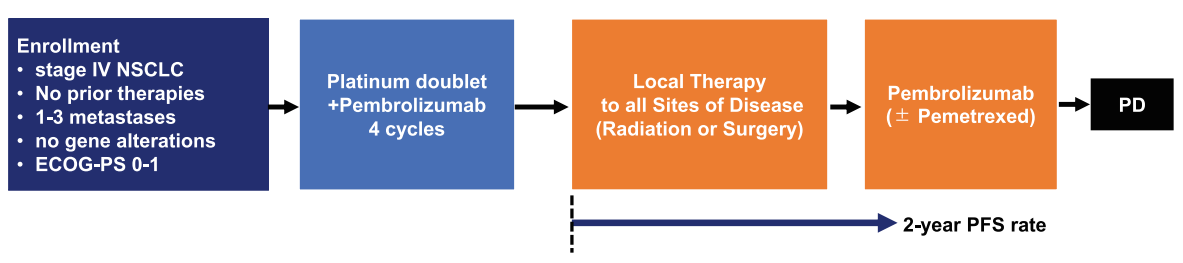

Primary endpoint : PFS rate at twenty-four months from start of local therapy

Secondary endpoint : Safety, ORR (Platinum doublet +Pembrolizumab), PFS and OS from start of local therapy, PFS and OS from enrollment

Fig. 1 ECOG, Eastern Cooperative Oncology Group; PS, performance status; PD, progressive disease; ORR, objective response rate; PFS, progression-free survival; OS, overall survival 
patient and the status of the tumour, including the tumour size and location, by a multidisciplinary tumour board. LAT is defined as radiotherapy to all sites of disease or as surgery for thoracic lesions including the primary site, regional lymph nodes, or pulmonary metastases.

Maintenance therapy will start within 42 days of the last day of LAT. Patients with non-squamous histology will receive pembrolizumab $(200 \mathrm{mg} /$ body $)+$ pemetrexed $\left(500 \mathrm{mg} / \mathrm{m}^{2}\right)$ by intravenous infusion on the first day. Patients with squamous histology will receive pembrolizumab ( $200 \mathrm{mg} /$ body) by intravenous infusion on the first day.

Tumor assessment will be conducted at baseline, every 6 weeks during the induction therapy, before LAT and every 9 weeks during the maintenance therapy. Tumor response will be evaluated in accordance with the Response Evaluation Criteria in Solid Tumors (RECIST, version 1.1). The prescribed adverse events and any severe adverse events will be recorded on the basis of the National Cancer Institute Common Terminology Criteria for Adverse Events (version 5.0). Concomitant use of anticancer drugs (including molecular targeted drugs and immune checkpoint inhibitors) that may affect protocol treatment is prohibited in this study. There is no special compensation for enrolled patients in this study.

\section{Data collection and monitoring}

All data related to this study is collected through the electric data capturing system throughout enrolment period and follow-up period. The data center of West Japan Oncology Group (WJOG) is supposed to take responsibility for management of data in this study. If the protocol of this study is amended, the WJOG data center will promptly notify the principal investigators at each site. The auditing is planned to conduct in accordance with regulations of WJOG.

The role responsibility of data monitoring committee (DMC) is to review efficacy and safety of this study independently from the investigators. The DMC review reports of severe adverse events from researchers and monitoring reports from the data center. The DMC comprised from four medical doctors who are independent from this study.

\section{Statistical analysis}

The primary endpoint is to evaluate the PFS rate of 24 months from the initiation of LAT. The 24-month PFS rate could be a reliable surrogate marker for the fiveyear survival rate in locally advanced NSCLC patients [13]. A phase II trial for oligometastatic NSCLC patients (defined by the investigators as 1-3 metastases) who received LAT after standard systemic therapy showed that the median PFS was 11.9 months and the 24-month PFS rate was approximately $25 \%$ [5]. Therefore, we set the threshold of the 24-month PFS rate at $25 \%$. In a previous phase II trial of oligometastatic NSCLC patients, the 24month PFS rate was approximately $50 \%$ following treatment with pembrolizumab after LAT, and over half the trial participants were previously treated with systemic therapy [12]. Thus, we expect a 24-month PFS rate of $60 \%$ should the trial population increase by a further $10 \%$. Under the current threshold and expected values, 17 patients receiving LAT would be required to achieve $80 \%$ power and a lower limit higher than $25 \%$ for the confidence interval of PFS rate. In a previous phase II trial, approximately $30 \%$ of the enrolled patients did not receive $\mathrm{LAT}$, owing to disease progression during induction systemic therapy $[5,6]$. Therefore, 30 patients who start induction therapy will initially be included, considering the possibility of patients dropping out. No interim analysis of this study will be performed.

\section{Discussion}

This phase II study aims to evaluate the efficacy of LAT at all sites of the disease following standard platinum doublet chemotherapy and pembrolizumab therapy in patients with synchronous oligometastatic NSCLC. LAT at all sites of the disease would inherently reduce the overall tumour burden, and this could promote $\mathrm{T}$ cell reinvigoration to enhance the efficacy of pembrolizumab [9-11], not just provide local control [2]. The ongoing phase III study might establish LAT at all disease sites following standard platinum doublet chemotherapy as a new standard therapy in patients with oligometastatic NSCLC [14]. This study will provide novel data on the efficacy and safety profile of the combination of LAT and chemotherapy plus immune-checkpoint inhibitors in patients with synchronous oligometastatic NSCLC.

\section{Conclusion}

If the results of this study meet the primary endpoint, we will recommend that the integrated strategy of LAT at all sites of disease following chemotherapy and pembrolizumab for patients with synchronous oligometastatic NSCLC be assessed further in more extensive phase III studies.

Abbreviations

LAT: Local ablative therapy; NSCLC: Non-small cell lung cancer; OS: Overall survival; PD: Progressive disease; PFS: Progression-free survival; PD-

1: Programmed cell death 1

\section{Acknowledgements}

We thank the data managers and other support staff of West Japan Oncology Group, especially Shinichiro Nakamura and Seiko Tanaka.

\section{Authors' contributions}

TM, HK, HH, YO, YC, KH, TO, TS, KW, KI, TU, SS, YK, YN, KN and NY contributed to the design of the study. $\mathrm{TM}, \mathrm{HK}, \mathrm{HH}$ and $\mathrm{YO}$ are the principal investigators 
of the study. TM, HK, HH, YO, KH, KW, TU, SS and KN will be involved in participant recruitment. $Y C$ was responsible for statistical analysis. All authors have read and approved the manuscript.

\section{Funding}

This study did not receive funding.

\section{Availability of data and materials}

Not applicable.

\section{Declarations}

\section{Ethics approval and consent to participate}

The WJOG $11118 \mathrm{~L}$ study is being conducted in compliance with the principles of the Declaration of Helsinki, and it was approved by the central review board of Shizuoka Cancer center. Written informed consent is obtained from all participants.

\section{Consent for publication}

Not applicable.

\section{Competing interests}

Dr. Kenmotsu reports grants and personal fees from AstraZeneca K.K., grants and personal fees from Chugai Pharmaceutical Co, Ltd., personal fees from Ono Pharmaceutical Co, Ltd., grants and personal fees from Boehringer Ingelheim, personal fees from Eli Lilly K. K, personal fees from Kyowa Hakko Kirin Co., Ltd., personal fees from Bristol-Myers Squibb, personal fees from MSD, grants and personal fees from Novartis Pharma K.K., grants and personal fees from Daiichi-Sankyo Co., Ltd., personal fees from Pfizer, and personal fees from Taiho Pharma, outside the submitted work. Dr. Harada reports personal fees from Daiichi Sankyo pharmaceutical Co., during the conduct of the study; personal fees from Daiichi Sankyo pharmaceutical co., personal fees from AstraZneca pharmaceutical co., personal fees from Brain labo co., personal fees from Chugai Pharmaceutical Co., grants from Japan Agency for Medical Research and Development, grants from The National Cancer Center Research and Development fund, outside the submitted work.

Dr. Chiba reports personal fees from Chugai Pharmaceutical Co., Ltd., outside the submitted work.

Dr. Haratani reports personal fees from AS ONE Corporation, grants and personal fees from AstraZeneca K.K., personal fees from Bristol-Myers Squibb Co. Ltd., personal fees from Chugai Pharmaceutical Co. Ltd., grants and personal fees from MSD K.K. 2018/11, personal fees from Ono Pharmaceutical Co. Ltd., and personal fees from Pfizer Japan Inc., outside the submitted work.

Dr. Sakamoto reports personal fees from AstraZeneca K.K., personal fees from Chugai Pharmaceutical Co., Ltd., personal fees from Merck KGaA, personal fees from Eli Lilly Japan K.K., personal fees from Novartis Pharma K.K., and personal fees from Kyowa Kirin Co., Ltd., outside the submitted work. Dr. Wakuda reports grants and personal fees from Chugai Pharmaceutical Co., Ltd., personal fees from Taiho Pharmaceutical, personal fees from Boehringer Ingelheim, personal fees from Eli Lilly K.K., personal fees from Ono Pharmaceutical, personal fees from MSD, grants from Novartis, grants from AbbVie, grants and personal fees from AstraZeneca, outside the submitted work.

Dr. Ito reports personal fees from Boehringer Ingelheim, personal fees from AstraZeneca, personal fees from Pfizer, Eli Lilly, personal fees from Chugai Pharmaceutical, personal fees from Merk Sharp \& Dohme (MSD), personal fees from Ono Pharmaceutical, personal fees from Taiho Pharmaceutical, during the conduct of the study.

Dr. Uemura reports personal fees from Chugai Pharmaceutical Co., Ltd., outside the submitted work.

Dr. Kogure reports personal fees from AstraZeneca K.K., Chugai

Pharmaceutical Co, Ltd., Eli Lilly K. K, and Boehringer Ingelheim., grants and personal fees from MSD, which are unrelated to the submitted work. Dr. Nakagawa reports grants and personal fees from AstraZeneca K.K., grants and personal fees from Astellas Pharma Inc., grants and personal fees from MSD K.K., grants, personal fees and other from Ono Pharmaceutical Co., Ltd., grants and personal fees from Nippon Boehringer Ingelheim Co., Ltd., grants and personal fees from Novartis Pharma K.K., grants, personal fees and other from Pfizer Japan Inc., grants and personal fees from Bristol-Myers Squibb Company, grants, personal fees and other from Eli Lilly Japan K.K., grants and personal fees from Chugai Pharmaceutical Co., Ltd., grants and personal fees from Daiichi Sankyo Co., Ltd., grants and personal fees from Merck Serono Co., Ltd./ Merck Biopharma Co., Ltd., during the conduct of the study; personal fees from Clinical Trial Co., Ltd., personal fees from MEDICUS SHUPPAN, Publishers Co., Ltd., personal fees from Care Net, Inc., personal fees from Reno. Medical K.K., personal fees and other from KYORIN Pharmaceutical Co., Ltd., personal fees from Medical Review Co., Ltd., personal fees from Roche Diagnostics K.K., personal fees from Bayer Yakuhin, Ltd., personal fees from Medical Mobile Communications co., Ltd., personal fees from $3 \mathrm{H}$ Clinical Trial Inc., personal fees from Nichi-lko Pharmaceutical Co., Ltd., grants, personal fees and other from Takeda Pharmaceutical Co., Ltd., grants and personal fees from Taiho Pharmaceutical Co., Ltd., grants and personal fees from SymBio Pharmaceuticals Limited., personal fees from NANZANDO Co., Ltd. personal fees from YODOSHA CO., LTD., personal fees from Nikkei Business Publications, Inc., personal fees from Thermo Fisher Scientific K.K., personal fees from YOMIURI TELECASTING CORPORATION., personal fees from Nippon Kayaku Co., Ltd., grants and personal fees from AbbVie Inc., grants from inVentiv Health Japan, grants from ICON Japan K.K., grants from GRITSONE ONCOLOGYINC, grants from PAREXEL International Corp., grants from Kissei Pharmaceutical Co., Ltd., grants from EPS Corporation., grants from Syneos Health., grants from Pfizer R\&D Japan G.K., grants from A2 Healthcare Corp., grants from Quintiles Inc. / IQVIA Services JAPAN K.K., grants from EP-CRSU CO., LTD., grants from Linical Co., Ltd., grants from Eisai Co., Ltd., grants from CMIC Shift Zero K.K., grants from Kyowa Hakko Kirin Co., Ltd., grants from Bayer Yakuhin, Ltd., grants from EPS International Co., Ltd..,, grants from Otsuka Pharmaceutical Co., Ltd., outside the submitted work.

Dr. Yamamoto reports grants and personal fees from MSD K.K., grants and personal fees from AstraZeneca, grants and personal fees from ONO PHARMACEUTICAL CO., LTD., personal fees from Thermo Fisher Scientific, grants and personal fees from Daiichi Sankyo Co., Ltd., grants and personal fees from TAIHO PHARMACEUTICAL CO., LTD., grants and personal fees from Takeda Pharmaceutical CO., LTD., grants and personal fees from Chugai Pharmaceutical Co., LTD., grants and personal fees from Eli Lilly Japan K.K., grants and personal fees from Boehringer Ingelheim, grants and personal fees from Novartis, grants and personal fees from Pfizer Inc., personal fees from Bristol-Myers Squibb, personal fees from Life Technologies Japan Ltd., personal fees from NIPPON KAYAKU, personal fees from Merk Biopharma, grants from Astellas Pharma Inc., grants from TSUMURA \& CO., grants from SHIONOGI Co., Ltd., grants from AbbVie G.K., grants from Amgen Inc., grants from KYORIN Pharmaceutical Co., Ltd., grants from Eisai Co., Ltd., grants from TERUMO CORPORATION, grants from Toppan Printing Co., Ltd., grants from $\mathrm{TOSOH}$, outside the submitted work.

\section{Author details}

${ }^{1}$ Division of Thoracic Oncology, Shizuoka Cancer Center, 1007

Shimonagakubo, Nagaizumi-cho, Sunto-gun, Shizuoka 411-8777, Japan.

${ }^{2}$ Department of Respiratory Medicine, Juntendo University Graduate School of Medicine, Tokyo, Japan. ${ }^{3}$ Radiation and Proton Therapy Center, Shizuoka Cancer Center, Shizuoka, Japan. ${ }^{4}$ Division of Thoracic Surgery, Shizuoka Cancer Center, Shizuoka, Japan. ${ }^{5}$ Clinical Research Center, Kindai University Hospital, Osaka-sayama, Japan. ${ }^{6}$ Department of Medical Oncology, Kindai University Faculty of Medicine, Osaka-sayama, Japan. ${ }^{7}$ Department of Internal Medicine, Division of Medical Oncology \& Respiratory Medicine, Shimane University Faculty of Medicine, Izumo, Japan. ${ }^{8}$ Division of Respiratory Medicine and Rheumatology, Department of Multidisciplinary Internal Medicine, Tottori University, Yonago city, Japan. ${ }^{9}$ Respiratory Centor, Matsusaka Municipal Hospital, Matsusaka, Japan. ${ }^{10}$ Department of Respiratory Medicine, Allergy and Clinical Immunology, Nagoya City University Graduate School of Medical Sciences, Nagoya, Japan. " Department of Respiratory Medicine, Kumamoto University Hospital, Kumamoto, Japan. ${ }^{12}$ Department of Respiratory Medicine, National Hospital Organization Nagoya Medical Center, Nagoya, Japan. ${ }^{13}$ Department of Radiation Oncology, Kindai University Faculty of Medicine, Osaka-sayama, Japan. ${ }^{14}$ Internal Medicine III, Wakayama Medical University, Wakayama, Japan.

\section{Received: 4 February 2021 Accepted: 8 October 2021}

Published online: 18 October 2021

\section{References}

1. Hellman S, Weichselbaum RR. Oligometastases. J Clin Oncol. 1995;13(1):810. https://doi.org/10.1200/JCO.1995.13.1.8. 
2. Kim C, Hoang CD, Kesarwala AH, Schrump DS, Guha U, Rajan A. Role of local ablative therapy in patients with oligometastatic and oligoprogressive non-small cell lung cancer. J Thorac Oncol. 2017;12(2):179-93. https://doi. org/10.1016/j.jtho.2016.10.012.

3. Giaj-Levra N, Giaj-Levra M, Durieux V, Novello S, Besse B, Hasan B, et al. Defining synchronous oligometastatic non-small cell lung cancer: a systematic review. J Thorac Oncol. 2019;14(12):2053-61. https://doi.org/10.1 016/j.jtho.2019.05.037.

4. Gomez DR, Tang C, Zhang J, Blumenschein GR Jr, Hernandez M, Lee JJ, et al. Local consolidative therapy vs. maintenance therapy or observation for patients with oligometastatic non-small-cell lung cancer: long-term results of a multi-institutional, phase II, randomized study. J Clin Oncol. 2019;37(18):1558-65. https://doi.org/10.1200/jco.19.00201.

5. Gomez DR, Blumenschein GR, Lee JJ, et al. Local consolidative therapy versus maintenance therapy or observation for patients with oligometastatic non-small-cell lung cancer without progression after firstline systemic therapy: a multicentre, randomised, controlled, phase 2 study. Lancet Oncol. 2016;17(12):1672-82. https://doi.org/10.1016/s1470-2045(16)3 0532-0.

6. lyengar P, Wardak Z, Gerber DE, Tumati V, Ahn C, Hughes RS, et al. Consolidative radiotherapy for limited metastatic non-small-cell lung cancer: a phase 2 randomized clinical trial. JAMA Oncol. 2018;4(1): e173501. https://doi.org/10.1001/jamaoncol.2017.3501.

7. Paz-Ares L, Luft A, Vicente D, Tafreshi A, Gümüs M, Mazières J, et al. Pembrolizumab plus chemotherapy for squamous non-small-cell lung cancer. N Engl J Med. 2018;379(21):2040-51. https://doi.org/10.1056/ nejmoa1810865.

8. Gandhi L, Rodríguez-Abreu D, Gadgeel S, Esteban E, Felip E, de Angelis F, et al. Pembrolizumab plus chemotherapy in metastatic non-small-cell lung cancer. N Engl J Med. 2018;378(22):2078-92. https://doi.org/10.1056/ NEJMoa1801005.

9. Miyawaki T, Kenmotsu H, Mori K, Miyawaki E, Mamesaya N, Kawamura T, et al. Association between clinical tumor burden and efficacy of immune checkpoint inhibitor monotherapy for advanced non-small-cell lung cancer. Clin Lung Cancer. 2020;21(5):e405-14. https://doi.org/10.1016/j.cllc.2020.02. 012.

10. Huang AC, Postow MA, Orlowski RJ, Mick R, Bengsch B, Manne S, et al. T-cell invigoration to tumour burden ratio associated with anti-PD-1 response. Nature. 2017:545(7652):60-5. https://doi.org/10.1038/nature22079.

11. Guisier F, Cousse S, Jeanvoine M, Thiberville L, Salaun M. A rationale for surgical debulking to improve anti-PD1 therapy outcome in non small cell lung cancer. Sci Rep. 2019;9(1):16902. https://doi.org/10.1038/s41598-019-52 913-z.

12. Bauml JM, Mick R, Ciunci C, Aggarwal C, Davis C, Evans T, et al, Pembrolizumab after completion of locally ablative therapy for oligometastatic non-small cell lung cancer: a phase 2 trial. JAMA Oncol. 2019;5(9):1283-90. https://doi.org/10.1001/jamaoncol.2019.1449.

13. Akamatsu H, Mori K, Naito T, Imai H, Ono A, Shukuya T, et al. Progressionfree survival at 2 years is a reliable surrogate marker for the 5 -year survival rate in patients with locally advanced non-small cell lung cancer treated with chemoradiotherapy. BMC Cancer. 2014;14(1):18. https://doi.org/10.11 86/1471-2407-14-18

14. Conibear J, Chia B, Ngai Y, Bates AT, Counsell N, Patel R, et al. Study protocol for the SARON trial: a multicentre, randomised controlled phase III trial comparing the addition of stereotactic ablative radiotherapy and radical radiotherapy with standard chemotherapy alone for oligometastatic non-small cell lung cancer. BMJ Open. 2018;8(4):e020690. https://doi.org/1 0.1136/bmjopen-2017-020690.

\section{Publisher's Note}

Springer Nature remains neutral with regard to jurisdictional claims in published maps and institutional affiliations.

Ready to submit your research? Choose BMC and benefit from:

- fast, convenient online submission

- thorough peer review by experienced researchers in your field

- rapid publication on acceptance

- support for research data, including large and complex data types

- gold Open Access which fosters wider collaboration and increased citations

- maximum visibility for your research: over $100 \mathrm{M}$ website views per year

At $\mathrm{BMC}$, research is always in progress.

Learn more biomedcentral.com/submissions 